\title{
0261 USING LAY HOME EDUCATORS TO PROVIDE SAFETY MESSAGES IN DALLAS, TX, USA
}

S Stephens-Stidham*, J Trudeau, G Istre, C Weir, U Johnson, R Anderson Correspondence: Injury Prevention Center of Greater Dallas, 5000 Harry Hines Blvd. Dallas, TX 75235, USA

10.1136/ip.2010.029215.261

Background Home visitation programs designed to decrease the risk of child maltreatment are well supported by research. However, there is a lack of research on the efficacy of home visitation programs conducted by lay educators. To examine if lay educators could provide education that impacts specific behaviour changes, the Injury Prevention Center of Greater Dallas partnered with the Texas Home Instruction for Parents of Preschool Youngsters to implement a residential fire and scald burn prevention project.

Methods The educators were randomly assigned into intervention and comparison groups. Pre intervention home assessments were conducted in both the intervention and comparison groups. Educators in the intervention group provided literature and one-time verbal education to participants on smoke detectors and proper hot water temperature. Educators in the comparison group only provided written literature. The post intervention assessments were conducted 2 months following the preassessment.

Results The prevalence of smoke detectors increased in both the comparison and intervention groups (intervention $13 \%$ increase; comparison $11 \%$ increase). In the intervention group, $14 \%$ decreased hot water temperature to at or below 120 degrees. Among the comparison group, 6\% decreased hot water temperature to at or below 120 degrees.

Conclusion There were limitations in the study and a second phase will be implemented. However, the program appears to be a cost-effective method to providing safety messages to families. 